\title{
Recursive distribution method for probabilistic fracture mechanics
}

\author{
Akiba, $\mathrm{H}^{1}$, Yoshimura, $\mathbf{S}{ }^{2}$, Yagawa, $\mathrm{G}^{2}$ \\ 1) Allied Engineering Corporation, Tokyo, Japan \\ 2) University of Tokyo, Tokyo, Japan
}

ABSTRACT This paper proposes a new method for Probabilistic Fracture Mechanics (PFM). The present method gives a recursive formula for a joint probability distribution function of crack geometry, which is obtained from random variables of the initial crack geometry and a deterministic time-evolution law of the variables. A numerical example of Light Water Reactor (LWR)'s piping is solved by the present method, and the results are compared with those of the Monte Carlo (MC) method. It is clearly shown that both results agree sufficiently well, while CPU time of the present method is remarkably short.

\section{INTRODUCTION}

Probabilistic Fracture Mechanics (PFM) has been playing an important role in the study on reliability of nuclear power plants. The basic idea of PFM is to give random fluctuation to the initial crack geometry_-initial crack depth and aspect ratio, and to assume a crack growth law as a deterministic process ${ }^{[3]}$. Other random fluctuations are omitted at the first step of the development of the present method.

The Monte Carlo (MC) method is used in many cases as a method for multiple integrals. The theoretical basis of convergence of the method depends on the law of large numbers and the central limit theorem in the probability theory. This is basically inefficient.

We propose here a deterministic numerical method for problems under the following general situation: (1) random variables which prescribe an initial state are given, and (2) a timeevolution law of the variables is assumed to be a deterministic discrete process represented by explicitly given functions. This process can also be a discretized solution of a differential equation. We construct a recursive formula for a probability measure induced by a joint distribution function of the variables.

This method is applied to PFM. A numerical example of a LWR piping problem is solved, and the results are compared with those obtained by the MC method. It is clearly demonstrated that the present method is a potential tool for PFM.

\section{RECURSIVE FORMULA FOR DISTRIBUTION FUNCTION}

Let $N$ be a positive integer, and let $\Phi$ be a map from $\mathbf{R}^{N}$ into itself, where $\mathbf{R}^{N}$ is the $N$-fold direct product of the real line $\mathbf{R}$. Denote

$$
\Phi\left(x_{1}, \cdots, x_{N}\right)=\left(\Phi_{1}\left(x_{1}, \cdots, x_{N}\right), \cdots, \Phi_{N}\left(x_{1}, \cdots, x_{N}\right)\right)
$$

Let $X=\left(X_{1}, \cdots, X_{N}\right)$ be a $\mathbf{R}^{N}$-valued random vector, then $\Phi\left(X_{1}, \cdots, X_{N}\right)$ is also a random vector.

Now, let $\left(X_{1}^{0}, \cdots, X_{N}^{0}\right)$ be a random vector, which prescribes the initial state, whose 
distribution function is

$$
H^{0}\left(x_{1}, \cdots, x_{N}\right)=\mathbf{P}\left(X_{1}^{0} \leq x_{1}, \cdots, X_{N}^{0} \leq x_{N}\right) .
$$

The time-evolution laws of the variables are assumed to be given by deterministic, and in general, time dependent relations as

$$
\left\{\begin{array}{c}
X_{1}^{\mathbf{n}+1}=\Phi_{1}^{n}\left(X_{1}^{n}, \cdots, X_{N}^{n}\right) \\
\cdots \\
X_{N}^{n+1}=\Phi_{N}^{n}\left(X_{1}^{n}, \cdots, X_{N}^{n}\right)
\end{array}\right.
$$

or

$$
X^{n+1}=\Phi^{n}\left(X^{n}\right)
$$

where $n$ denotes a discrete time index. Our objective is to determine a recursive formula for the distribution function $H^{n}$ of $\left(X_{1}^{n}, \cdots, X_{N}^{n}\right)$. We give some preparatory comments regarding the measure theory.

Contrary to the fact that a set of random variables defines a joint distribution function, a function $H$ on the interval

$$
D=\left(a_{1}, b_{1}\right] \times \cdots \times\left(a_{N}, b_{N}\right],
$$

satisfying the following conditions is called a distribution function, and there exists a set of random variables with the distribution, which assumes value in $D$.

(i) The following two equations are satisfied:

$$
\begin{aligned}
& H\left(b_{1}, \cdots, b_{N}\right)=1 \\
& H\left(a_{1}, \cdots, a_{N}\right)=0
\end{aligned}
$$

(ii) Let the function $H$ extend to the set of all intervals in $D$ in the form

$$
I=\left(x_{1}^{(1)}, x_{1}^{(0)}\right] \times \cdots \times\left(x_{N}^{(1)}, x_{N}^{(0)}\right]
$$

by

$$
H(I)=\sum_{i_{1}=0}^{1} \cdots \sum_{i_{N}=0}^{1}(-1)^{i_{1}+\cdots+i_{N}} H\left(x_{1}^{\left(i_{1}\right)}, \cdots, x_{N}^{\left(i_{N}\right)}\right) .
$$

Then, for all intervals $I \subset D$, we have

$$
H(I) \geq 0
$$

(iii) For $j=1,2, \cdots$, we have

$$
\lim _{x_{j}^{(0)} \backslash x_{j}^{(1)}} H\left(\left(x_{1}^{(1)}, x_{1}^{(0)}\right] \times \cdots \times\left(x_{j}^{(1)}, x_{j}^{(0)}\right] \times \cdots \times\left(x_{N}^{(1)}, x_{N}^{(0)}\right]\right)=0
$$

A distribution function $H$ can be extended to a probability measure $H_{*}$ (a bounded Lebesgue-Stieltjes measure) on the Lebesgue-Stieltjes field $\mathbf{M}$ induced by $H .{ }^{[8]}$

$H_{*}$ is induced from $H$ in the following way ${ }^{[2]}$ :

(1) Define the outer measure $H_{* *}$ by

$$
H_{* *}(A)=\inf \sum_{i=1}^{\infty} H\left(I_{i}\right)
$$


for any $A \subset D$, where $\left\{I_{i}\right\}$ is a family of intervals which satisfies

$$
A \subset \bigcup_{i=1}^{\infty} I_{i}
$$

and the infimum of the right hand side of the equation (12) is taken over all families $\left\{I_{i}\right\}$ satisfying (13). $H\left(I_{i}\right)$ in the right hand side of (12) is obtained by the formula (9).

(2) The Lebesgue-Stieltjes measurable subsets in $D$ are defined through the outer measure $H_{* *}$, according to the definition of measurability. All the measurable subsets constitute the Lebesgue-Stieltjes measurable field $\mathbf{M}$, which contains all the Borel subsets in $D$. The restriction of $H_{* *}$ to $\mathbf{M}$ is the Lebesgue-Stieltjes measure $H_{*} H_{*}$ is an extension of $H$. Therefore we use the same notation $H$ for this measure.

Lemma. Let $H$ be a Lebesgue-Stieltjes measure on $D$, and let $\Psi$ be a $C^{1}$-map on $D$ into itself. If $A$ in $D$ is a null set with respect to $H$, so is $\Psi(A)$. That is, if $H(A)=0$, then $H(\Psi(A))$ $=0$.

Now we proceed to the recursive formula:

Theorem. Let the initial joint distribution $H^{0}$ be given. Let the map $\Phi^{n}, n=1,2, \cdots$ be a $\mathrm{C}^{1}$-isomorphisms on $D$ into itself. The recursive formula is given by

$$
H^{n+1}(I)=H^{n}\left(\left(\Phi^{n}\right)^{-1}(I)\right)
$$

where $I$ is an interval in $D$ and $\left(\Phi^{n}\right)^{-1}(I)$ is the inverse image of $I$ under $\Phi^{n}$. $H^{n}$ in the right hand side should be interpreted as a probability measure.

Proof. We define a function $H$ on $D$ by

$$
H\left(x_{1}, \cdots, x_{N}\right)=H^{n}\left(\left(\Phi^{n}\right)^{-1}\left(\left(a_{1}, x_{1}\right] \times \cdots \times\left(a_{N}, x_{N}\right)\right),\right.
$$

using the right hand side of (14). We have to prove that $H$ is a distribution function on $D$. It is easy to obtain (i) and (ii). (iii) is followed by the above lemma. Accordingly, the function $H$ defines a probability measure as stated previously. Equation (14) is then apparent as follows:

$$
H^{n+1}(I)=\mathbf{P}\left(X^{n+1} \in I\right)=\mathbf{P}\left(\Phi^{n}\left(X^{n}\right) \in I\right)=\mathbf{P}\left(X^{n} \in\left(\Phi^{n}\right)^{-1}(I)\right)=H^{n}\left(\left(\Phi^{n}\right)^{-1}(I)\right) .
$$

It is not so simple to evaluate the right hand side of $(14)$, because the image $\left(\Phi^{n}\right)^{-1}(I)$ is distorted from the interval (rectangle) $I$. From the view point of application, the formula (9) gives an approximation method for the right hand side of (14), by partitioning the image $\left(\Phi^{n}\right)^{-1}(I)$ into a number of fine intervals.

\section{APPLICATION TO PFM}

A single crack is assumed to exist on the inner surface of a pipe in the circumferential direction. The crack is assumed to be semi-elliptical with a crack depth $a$ and a half length $b$ (See Fig.1). Let $\alpha=a / h$ and $\gamma=a / b$, where $h$ is a wall thickness, accordingly $0<\alpha \leq 1$. The aspect ratio $\gamma$ is assumed to be $0<\gamma \leq 1$. The time scale is divided into discrete time steps $n=0,1, \cdots$. As for a crack growth law, we employ the Paris-Erdogan equation ${ }^{[7]}$

$$
\left\{\begin{array}{l}
a^{n+1}=a^{n}+C\left(\Delta K_{\mathrm{A}}\left(a^{n}, b^{n}\right)\right)^{\nu} \Delta N \\
b^{n+1}=b^{n}+C\left(\Delta K_{\mathrm{B}}\left(a^{n}, b^{n}\right)\right)^{\nu} \Delta N
\end{array}\right.
$$

where $\Delta N$ is the number of applied stress cycles between two successive time steps, $C$ and $\nu$ are material constants, and $\Delta K_{\mathrm{A}}$ and $\Delta K_{\mathrm{B}}$ are the effective stress intensity factors defined by 


$$
\Delta K(a, b)=K(a, b)_{\max }-K(a, b)_{\min }
$$

In (18), the stress intensity factor $K$ represents $K_{\mathrm{A}}$ or $K_{\mathrm{B}}$ in the thickness or surface direction, respectively (See Fig.1). $K$ can be expressed by Newman-Raju's formula ${ }^{[6]}$.

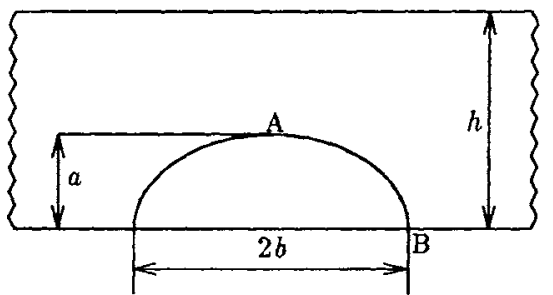

Fig.1 Assumed semi-elliptical surface crack

From (17), we obtain the time-evolution law of the crack geometry in the form of

$$
\left\{\begin{array}{l}
\Phi(x, y)=(\phi(x, y), \psi(x, y)) \\
\left(\alpha^{n+1}, \gamma^{n+1}\right)=\Phi\left(\alpha^{n}, \gamma^{n}\right),
\end{array}\right.
$$

where $\Phi$ is a deterministic mapping from the unit rectangle $\{0,1\} \times[0,1]$ into $\mathbf{R}^{2}$.

The initial crack geometry $\left(\alpha^{0}, \gamma^{0}\right)$ is taken as a random vector. $\alpha^{0}$ and $\gamma^{0}$ are assumed to be independent with the distribution functions $F(x)$ and $G(y)$, respectively. The joint distribution of the random vector $\left(\alpha^{0}, \gamma^{0}\right)$ is expressed as

$$
H^{0}(x, y)=F(x) G(y)
$$

The theorem in the previous section is applied to the present situation. Our goal is to evaluate time histories of leakage probabilities. The leakage criterion is assumed to be $\alpha>\alpha_{c}$, where $\alpha_{c}$ is a threshold value. The leakage probability is given by the marginal distribution

$$
p^{n}=\mathbf{P}\left(\alpha^{n}>\alpha_{c}\right)=1-H^{n}\left(\alpha_{c}, 1\right)
$$

\section{ANALYSIS OF LWR PIPING}

\subsection{ANALYSIS CONDITIONS AND DISCRETIZATION OF RECURSIVE FORMULA}

A pipe analyzed has an outer diameter of $660.4 \mathrm{~mm}$ and a thickness of $33.3 \mathrm{~mm}$. The material assumed is carbon steel, STS410. A design allowable stress $S_{n}$ is assumed to be $122.6 \mathrm{MPa}$ $\left(300^{\circ} \mathrm{C}\right)$, which is taken from Japanese MITI notification $501^{[10]^{n}}$. The crack growth parameters are $C=1.75 \times 10^{-10}, \nu=5.95$ if $\Delta K \leq 13.2\left[\mathrm{MPam}^{1 / 2}\right]$, otherwise $C=5.34 \times 10^{-6}, \nu=1.95$, which are taken from the reference crack growth laws of ferritic steels in water given in ASME Code Sec.XII ${ }^{[9]}$. Design loading conditions for LWR piping as shown in Table 1 are chosen ${ }^{[I I]}$. The threshold value of the leakage criterion of (16) is assumed to be $\alpha_{c}=0.8$.

The initial crack depth $a$ is assumed to have the Marshall distribution ${ }^{[5]}$, i.e. the exponential distribution with the parameter $\lambda=0.16[1 / \mathrm{mm}]$. The aspect ratio $b / a(=1 / \gamma)$ is assumed to be lognormally distributed with the median value $\mu_{0}=1.336$ and the logarithmic standard deviation $S_{0}=0.538^{[3]}$.

Discretization is determined through a geometric consideration for the inverse image $\Phi^{-1}([0, x] \times[0, y])$ in the recursive formula (14). Fig.2 shows an example of a determined partition of the inverse image. In order to clearly depict the distortion of the image, an extremely larger stress than in Table 1 is applied. A number of oblong rectangles are taken along $\alpha$ axis. We have to specify the numbers $M$ and $N$ of the partitions of $\alpha$ and $\gamma$ axes, respectively. 
Table 1 Loading conditions for pipe

\begin{tabular}{|c|cc|cc|c|}
\hline \multirow{2}{*}{ No } & \multicolumn{3}{|c|}{ Applied stress $\left(\times S_{m}\right)$} & \multirow{2}{*}{$\begin{array}{c}\text { Cycles } \\
\text { /year }\end{array}$} \\
\cline { 2 - 5 } & \multicolumn{2}{|c|}{ Tension } & \multicolumn{2}{c|}{ Bending } & \\
\cline { 2 - 5 } & $\max$ & $\min$ & $\max$ & $\min$ & \\
\hline 1 & 1.0 & 0.0 & 0.0 & 0.0 & 7 \\
2 & 1.5 & 0.4 & 0.0 & 0.0 & 18 \\
3 & 1.0 & 0.75 & 0.0 & 0.0 & 320 \\
4 & 0.0 & 0.0 & 1.0 & -1.0 & 8 \\
5 & 0.0 & 0.0 & 0.5 & -0.5 & 16 \\
6 & 0.0 & 0.0 & 0.1 & -0.1 & 330 \\
\hline
\end{tabular}

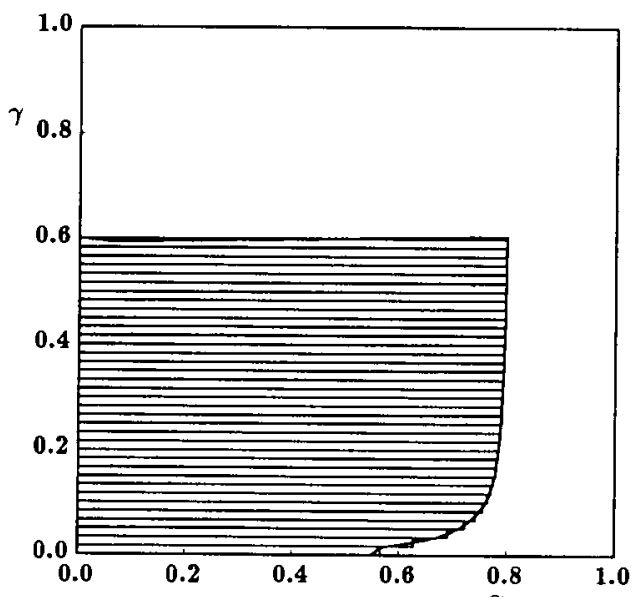

Fig.2 Partition of the image $\Phi^{-1}([0, x] \times[0, y]) \stackrel{\alpha}{(x=0.8, y=0.6, L=60)}$

\subsection{ANALYSIS RESULTS}

Now we solve the PFM problem stated in the above section. The computer used is SUN Sparc Station 10. The numbers $M$ and $N$ are varied ranging from 50 to 300 .

For comparison, the same problem is calculated using the MC method. The present MC method uses the stratified sampling technique. The number of cells is taken to be 48 . The number of samples from each cell is taken to be 3,000 and 10,000. Since the results are almost the same, we regard the two cases as the reference solution.

Results and the CPU time are shown in Table 2 and Fig.3. It can be seen that (1) the results of the present method are stable under different discretizations; and that (2) the results of the present method and the MC are very close at 1 or 2 years, then the former results are gradually getting larger than the reference solution. The result (1) means the stability of the calculation and the capability of the use of a coarse partition. The reason of the discrepancy might be attributed to the partition of the image by rectangles. A further investigation is still needed.

As for CPU time, the present method shows remarkable performance. The case $50 \times 50$ partition took only 2 minutes, while the MC method spent several hours.

\section{CONCLUSIONS}

A new deterministic method for PFM was proposed. The present method is constructed by a general form of recursive formula for the joint distribution function of crack geometry. It was applied to a LWR's piping problem. The results showed good agreement with those of the MC method. The CPU time of the present method was only 2 minutes for the calculation, while 
the MC method spent several hours.

Table 2 Results and CPU times

\begin{tabular}{|c|c|c|c|c|c|}
\hline \multirow{2}{*}{ year } & \multicolumn{3}{|c|}{ Deterministic } & \multicolumn{2}{c|}{ MC } \\
\cline { 2 - 6 } & $300 \times 300^{-}$ & $100 \times 100$ & $\mathbf{5 0 \times 5 0}$ & $10000^{*+}$ & 3000 \\
\hline 0 & $9.2797 \times 10^{-3}$ & $9.2797 \times 10^{-3}$ & $9.2797 \times 10^{-3}$ & $9.2797 \times 10^{-3}$ & $9.2797 \times 10^{-3}$ \\
1 & $9.7151 \times 10^{-3}$ & $9.7210 \times 10^{-3}$ & $9.7315 \times 10^{-3}$ & $9.7126 \times 10^{-3}$ & $9.8597 \times 10^{-3}$ \\
2 & $1.0373 \times 10^{-2}$ & $1.0387 \times 10^{-2}$ & $1.0414 \times 10^{-2}$ & $1.0361 \times 10^{-2}$ & $1.0498 \times 10^{-2}$ \\
3 & $1.1050 \times 10^{-2}$ & $1.1074 \times 10^{-2}$ & $1.1116 \times 10^{-2}$ & $1.1017 \times 10^{-2}$ & $1.1130 \times 10^{-2}$ \\
10 & $1.5921 \times 10^{-2}$ & $1.6015 \times 10^{-2}$ & $1.6179 \times 10^{-2}$ & $1.5788 \times 10^{-2}$ & $1.5798 \times 10^{-2}$ \\
20 & $2.4109 \times 10^{-2}$ & $2.4345 \times 10^{-2}$ & $2.4742 \times 10^{-2}$ & $2.3099 \times 10^{-2}$ & $2.3115 \times 10^{-2}$ \\
40 & $4.4862 \times 10^{-2}$ & $4.5494 \times 10^{-2}$ & $4.6539 \times 10^{-2}$ & $4.0434 \times 10^{-2}$ & $4.0447 \times 10^{-2}$ \\
80 & $1.0607 \times 10^{-1}$ & $1.0787 \times 10^{-1}$ & $1.1077 \times 10^{-1}$ & $8.6776 \times 10^{-2}$ & $8.7301 \times 10^{-2}$ \\
\hline CPU & $1.1 \mathrm{hr}$ & $8.1 \mathrm{~min}$ & $1.9 \mathrm{~min}$ & $7.9 \mathrm{hr}$ & $2.3 \mathrm{hr}$ \\
\hline
\end{tabular}

"the numbers indicate $M \times N ;{ }^{* *}$ the numbers indicate the number of samples taken trom each cell

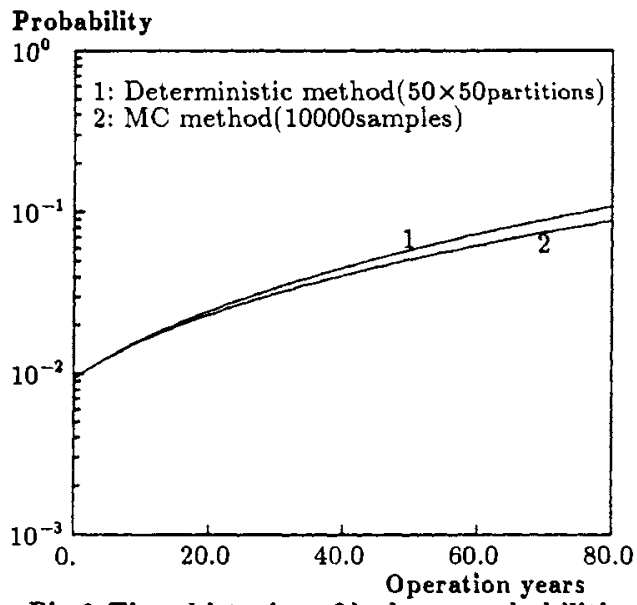

Fig.3 Time histories of leakage probabilities

\section{REFERENCES}

[1] Akiba, H., Yoshimura, S. and Yagawa, G., submitted for publication, Direct Analysis Method for Probabilistic Fracture Mechanics, Nuclear Engineering and Design.

[2] Halmos, P.R., 1974, Measure Theory, Springer-Verlag, New York.

[3] Harris, D. O., Lim, E. Y. and Dedhia, D. D., 1981, Probability of Pipe Fracture in the Primary Coolant Loop of a PWR Plant, NUREG/CR-2189.

[4] Hirsch, M. W., 1976, Differential Topology, Springer-Verlag, New York.

[5] Marshall, W., 1982, An Assessment of the Integrity of PWR Pressure Vessels, UKAEA.

[6] Newman Jr, J. C. and Raju, I. S., 1981, An Empirical Stress-Intensity Factor Equation for the Surface Crack, Engineering Fracture Mechanics, 15: 185-192.

[7] Paris, P. and Erdogan, F., 1963, A Critical Analysis of Crack Propagation Laws, Transactions of ASME, Journal of Basic Engineering, 85: 528-534.

[8] Wong, E., 1971, Stochastic Processes in Information and Dynamical Systems, McGraw-Hill Inc., New York.

[9] ASME, 1973, ASME Boiler \& Pressure Vessel Code, Section XI, Appendix A.

[10]Japanese MITI Notification 501, 1980.

[11]JSME, 1995, Final Report of RC111 Subcommittee on Probabilistic Fracture Mechanics, (in Japanese). 